\title{
Isolation and Characterization of a Class III Peroxidase cDNA from Cucumber under Salt Stress
}

\author{
Huai-Fu Fan', Wen Chen ${ }^{1}$, Zhou Yu, and Chang-Xia Du ${ }^{2}$ \\ The Key Laboratory for Quality Improvement of Agricultural Products of Zhejiang Province, School \\ of Agriculture and Food Science, Zhejiang Agriculture \& Forestry University, Lin'an 311300, P.R. \\ China
}

\begin{abstract}
AdDitional INDEX words. Cucumis sativus, salinity
Abstract. Salt stress reduces the fresh weight, dry weight, and relative growth rate of cucumber (Cucumis sativus) seedlings and results in serious quality loss in cucumber production. Our previous study indicated that the nettingassociated peroxidase (CsaNAPOD) protein in cucumber seedling roots was induced by salt stress. Here, we amplified the coding sequence of $C s a N A P O D$ from a cDNA isolated from the roots of cucumber seedlings. Sequence analysis indicated that the coding sequence of CsaNAPOD is $1035 \mathrm{bp}$, encoding a deduced protein of 344 amino acids, with a predicated molecular weight of $37.2 \mathrm{kD}$ and theoretical isoelectric point of 5.64. The deduced amino acid sequence of $\mathrm{CsaNAPOD}$ showed high sequence similarity to peroxidases (PODs) from other plant species. Moreover, CsaNAPOD possesses the typical sequence structures of class III PODs and indicated that CsaNAPOD belongs to this subfamily. CsaNAPOD was highly expressed in the roots and was weakly expressed in the stems and leaves of cucumber seedlings. Salt stress significantly increased the expression of $C s a N A P O D$ in the leaves during the entire experimental period compared with the control, and the expression of $C s a N A P O D$ in roots was reduced at 6 hours and induced at 48 and 72 hours by salt treatment. In stems, the expression of $C s a N A P O D$ declined at 48 and 72 hours as a result of the salt treatment compared with the control. These results indicate that the expression of $C s a N A P O D$ responded to salt stress in cucumber seedlings, and the expression patterns under salt stress in different tissues were not identical. Our research suggests that CsaNAPOD may have potential function during the plant response to salt stress.
\end{abstract}

Peroxidases, which comprise an important class of oxidoreductases, are widely distributed in all living organisms. PODs use $\mathrm{H}_{2} \mathrm{O}_{2}$ as their electron acceptor and iron porphyrin as their prosthetic group to catalyze substrate oxidation (Valério et al., 2004; van Huystee, 1987).

Plant PODs belong to a superfamily that consists of three different classes of PODs (Smulevich et al., 2006). Class I, the intracellular POD called catalase-peroxidase, is found in plants, bacteria, fungi, and Archaea. The primary function of class I PODs in the cell involves the detoxification of excess $\mathrm{H}_{2} \mathrm{O}_{2}$ (Shigeoka et al., 2002). Class II contains extracellular PODs secreted by fungi such as lignin PODs and manganese PODs that are involved in the degradation of soil debris (Martinez et al., 2005). Class III includes the large family of secreted PODs in plants that are secreted into the cell wall or the surrounding medium and the vacuole. In the standard peroxidative cycle, these PODs catalyze the reduction of $\mathrm{H}_{2} \mathrm{O}_{2}$ using electrons from various donor molecules. Class III PODs have been implicated in various functions, including auxin catabolism and the biosynthesis of secondary metabolites; the defense against pathogen penetration, wounding, and other abiotic stresses; cell wall hardening through the immobilization and extension of matrix polysaccharide crosslinking; and lignin and suberin deposition (Cosio and Dunand, 2009; De Gara, 2004). Recently, class III PODs have also been considered as potentially important

Received for publication 4 Apr. 2014. Accepted for publication 19 May 2014. This work was supported by the Zhejiang Provincial Natural Science Foundation of China (No. Y3110308) and the National Natural Science Foundation of China (No. 31101539 and No. 31201658).

${ }^{1}$ These authors contributed equally to this work.

${ }^{2}$ Corresponding author. E-mail: changxiadu@zafu.edu.cn. components of plant signal transduction pathways (McInnis et al., 2005).

Genes encoding class III PODs belong to a large multigenic family with 73 members in Arabidopsis thaliana (Tognolli et al., 2002), 138 members in rice [Oryza sativa (Passardi et al., 2004)], and 173 members in the typical grass model [Brachypodium distachyon (International Brachypodium Initiative, 2010)]. However, the presence of multiple isoforms and the complexity of the physiological processes regulated by these enzymes create difficulty in understanding the unique function of a specific class III POD member in plant development and in adapting to the environment. Information regarding the expression pattern during different developmental stages in tissues and under stress conditions aids in the understanding of the unique role of a specific POD.

The expression of class III POD genes is organ-dependent and influenced by abiotic stresses. Class III PODs in A. thaliana, Sesbania rostrata, and Senecio squalidus show obvious organ specificity, suggesting a highly specific role in the corresponding organ (Cosio and Dunand, 2009). The expression of class III POD genes is regulated by various environmental factors, including drought (Csiszár et al., 2012; Mohammadi et al., 2008), aluminum stress (Kumari et al., 2008), air pollutants and ultraviolet radiation (Kim et al., 2007), wounding (Gerchikov et al., 2008), and damage from plant disease and pests (Ansari et al., 2007; Severino et al., 2012). However, to date, information regarding class III $P O D$ gene expression mediated by salt is very limited.

Cucumber is one of the most important cucurbit (Cucurbitaceae) vegetables worldwide and is sensitive to a saline environment. The physiological responses of cucumber seedlings to salt stress have been well characterized. Specifically, salt stress destroyed the steady-state balance of reactive oxygen species 
(A)

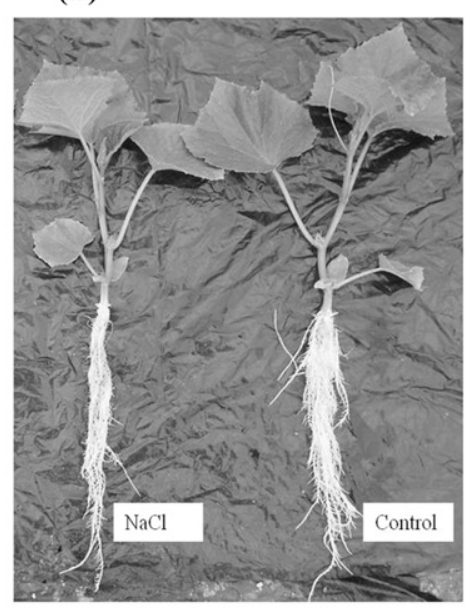

(C)

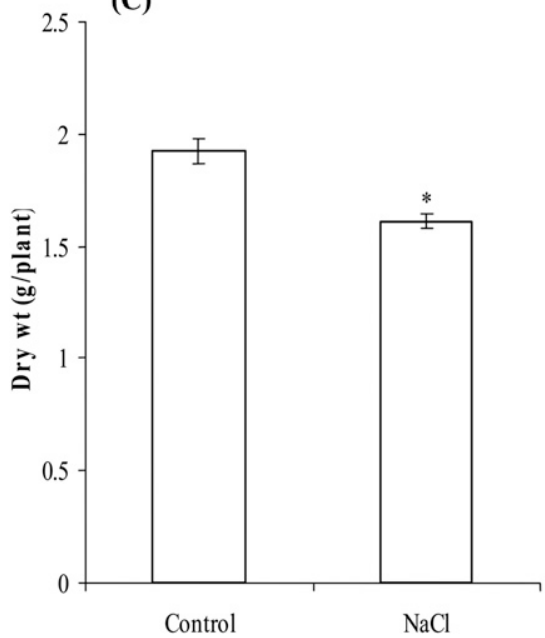

(B)

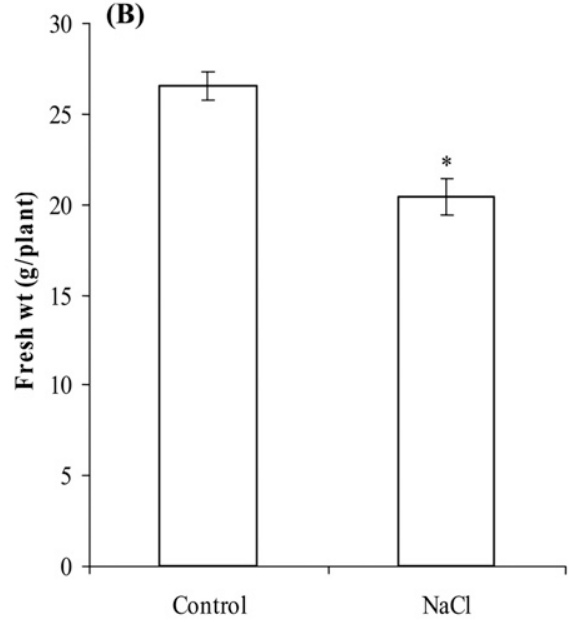

(D)

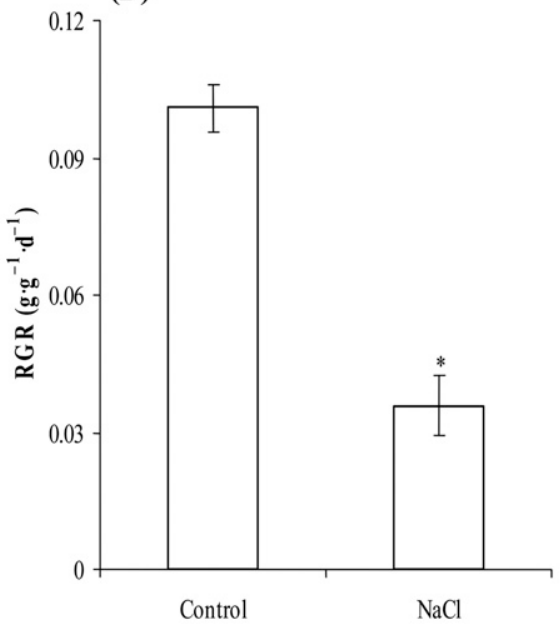

Fig. 1. The effect of salt stress on the growth of the cucumber seedlings after $96 \mathrm{~h}$ of treatment $(\mathrm{Control}=\mathrm{control}$ plants, $\mathrm{NaCl}=50 \mathrm{~mm} \mathrm{NaCl}$-treated plants). An image of cucumber seedlings treated with $50 \mathrm{~mm} \mathrm{NaCl} \mathrm{for} 96 \mathrm{~h}$ and control seedlings $(\mathbf{A})$. Fresh weight $(\mathbf{B})$, dry weight $(\mathbf{C})$, and relative growth rate [RGR (D)] were determined. Each histogram represents the mean value of six independent experiments, and the vertical bars indicate the SE $(\mathrm{n}=6)$. Asterisks indicate the significant differences calculated using the $t$ test $\left({ }^{*} P<0.05\right)$.

characteristics and designated the protein as CsaNAPOD. The expression pattern of CsaNAPOD in different cucumber seedling tissues over time under salt treatment was also investigated. The objective of the present research was to investigate whether CsaNPOD is regulated by salt stress at the transcriptional level.

\section{Materials and Methods}

Plant Materials and TREATMENT. The experiments were conducted in an environment-controlled greenhouse. Cucumber cultivar Jinchun 2 seeds were allowed to germinate in the dark in a thermostatically controlled chamber at 28 to $30{ }^{\circ} \mathrm{C}$ for $\approx 30 \mathrm{~h}$. The germinated seeds were sown in 50-cell plug trays containing peatmoss and perlite $(1: 1, \mathrm{v} / \mathrm{v})$ at $1200 \mu \mathrm{mol} \cdot \mathrm{m}^{-2} \cdot \mathrm{s}^{-1}$ photosynthetic photon flux and a 14/ $10 \mathrm{~h}\left(25\right.$ to $30 / 16$ to $\left.20^{\circ} \mathrm{C}\right)$ day/night regime. Relative aerial humidity fluctuated between $60 \%$ and $75 \%$. When the cotyledons expanded, the seedlings were supplied with half-strength Hoagland's nutrient solution [pH 6.4 to 6.6 , electrical conductivity (EC) 2.0 to 2.2 $\left.\mathrm{dS} \cdot \mathrm{m}^{-1}\right]$. After the full development of the second leaf of each plant, 12 seedlings of uniform size were transplanted into a plastic container $(51 \times 33 \times 20 \mathrm{~cm})$ filled with fullstrength Hoagland's nutrient solution. The nutrient solutions were kept at 20 to $25^{\circ} \mathrm{C}$ and continuously aerated using an air pump at an

(ROS) in cucumber seedlings, resulting in the loss of relative water content and net photosynthetic rates as well as a decrease in plant growth rates (Du et al., 2010a; Duan et al., 2008; Fan et al., 2012; Shi et al., 2007). Therefore, studies on salt stress are important to further understand the developmental mechanisms affected by salt for cucumber production.

In our previous study, to identify components of salt stress signaling, we analyzed the protein expression profiles of the roots of cucumber seedlings treated with $50 \mathrm{mmol} \mathrm{NaCl}$ for 3 $\mathrm{d}$ using two-dimensional gel electrophoresis, and we identified a few differentially expressed proteins using matrix-assisted laser desorption/ionization time-of-flight mass spectrometry and liquid chromatography electrospray ionization tandem mass spectrometry (Du et al., 2010b). We found that one of the identified proteins, named netting-associated peroxidase [NAPOD (high peptide coverage with netting-associated peroxidase in Cucumis melo)], was induced in cucumber seedling roots under salt stress and may be involved in the defense against stress in cucumber seedlings (Du et al., 2010b). In the present study, we further analyzed the protein sequence interval of 20 min to maintain the dissolved oxygen at 7.8 to $8.2 \mathrm{mg} \cdot \mathrm{L}^{-1}$. After $3 \mathrm{~d}$ of pre-culture, the treatments were started. The cucumber seedlings were treated as follows: 1) control plants were grown in Hoagland's solution; and 2) salt-treated plants were grown in Hoagland's solution plus $50 \mathrm{~mm} \mathrm{NaCl}$. $\mathrm{NaCl}$ was added directly into the nutrient solution at the beginning of the treatment, and the plastic containers were arranged in a completely randomized block design with three replicates for each treatment (for a total of six plastic containers, each with 12 seedlings).

Growth DETERMination. To understand the effects of salt treatment on cucumber development, we treated the precultured cucumber seedlings with $50 \mathrm{~mm} \mathrm{NaCl}$ for $96 \mathrm{~h}$. At $96 \mathrm{~h}$ after $\mathrm{NaCl}$ treatment, six seedlings were prepared and were washed with tap water two to three times, rinsed twice with distilled water, gently blotted dry with a paper towel, and weighed to determine the weight of the seedlings when fresh. After measuring the fresh weight, the samples were killed in an oven at $105^{\circ} \mathrm{C}$ for $15 \mathrm{~min}$, dried at $75^{\circ} \mathrm{C}$ for $72 \mathrm{~h}$, and the dry weight was measured. The relative growth rate was defined as 


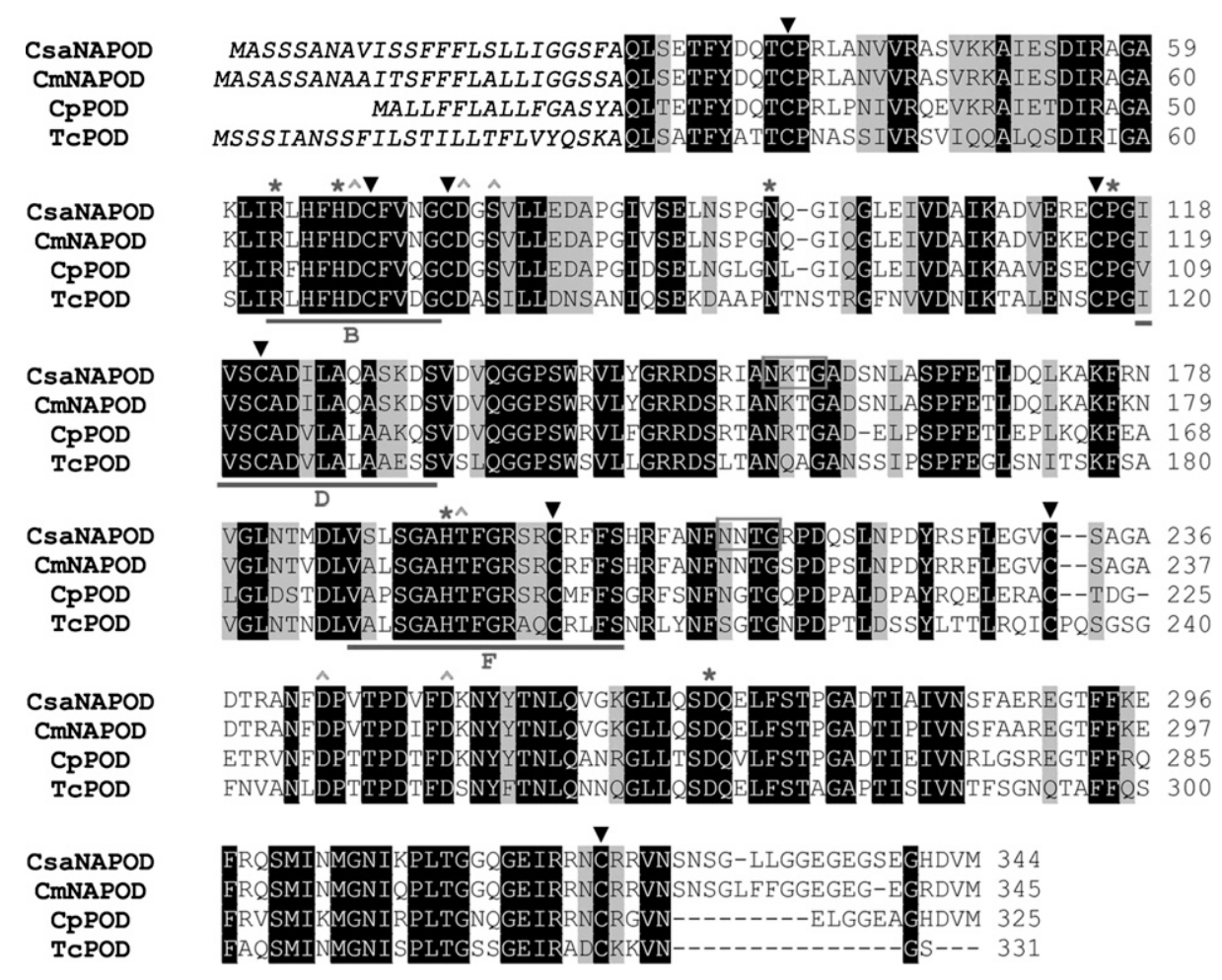

Fig. 2. The alignment of the predicted amino acid sequences of Cucumis sativus netting-associated peroxidase ( $C s a$ NAPOD) and three homologous peroxidases (PODs) with the highest similarity from different plant species. The accession numbers of the used amino acid sequences are as follows: C. sativus [Csa (XP_004151583.1)], Cucumis melo [Cm (AAR19041.1)], Cucurbita pepo [Cp (ABF68751.1)], and Theobroma cacao [Tc (EOY09519.1)]. The three horizontal red lines correspond to the conserved distal heme-binding domain (B), the proximal heme-binding domain $(\mathrm{F})$, and central conserved domains of unknown function (D). The predicted Nterminal signal peptide is italicized, the heme-binding sites are marked by a red asterisk, the calcium binding sites are marked by the inverted blue "V" symbol, and S-S-bridge-forming cysteines are noted by the inverted black triangle. The two green boxes indicate the putative glycosylation sites. collection, RNA extraction and cDNA synthesis were performed with three replicates and three experiments.

PCR AMPLIFICATION OF THE CSANAPOD CODING SEQUENCE. Using the Protein-BLAST tool in combination with the cucumber genomic database (Chinese Academy of Agricultural Sciences, 2009), we found only one protein, Csa023919, denoted also as POD 2 in $C$. sativus, with $100 \%$ identity to the CsaNAPOD amino acid sequence, indicating that CsaNAPOD and Csa023919 are the same protein. cDNA isolated from the roots of cucumber seedlings was used as the template for the PCR. The CsaNAPOD coding sequence was amplified corresponding to the coding sequence of Csa023919 in the cucumber genomic database. The primers used were as follows: forward, 5ATGGCCTCCTCCTCCGCC-3, reverse, 5-TTACATAACATCG TGGCCTTCTGAT-3. The PCR was conducted according to the following program: $94{ }^{\circ} \mathrm{C}$ for $5 \mathrm{~min}$ followed by 30 cycles of $30 \mathrm{~s}$ at $94{ }^{\circ} \mathrm{C}, 30 \mathrm{~s}$ at $60{ }^{\circ} \mathrm{C}, 60 \mathrm{~s}$ at $72{ }^{\circ} \mathrm{C}$, with a final extension step at $72{ }^{\circ} \mathrm{C}$ for $10 \mathrm{~min}$.

The PCR product was separated through a $1.2 \%$ agarose gel and purified with the Agarose Gel DNA Extraction Kit (TaKaRa Bio). The fragment was inserted into a pMD18-T vector (TaKaRa

the parameter $R G R$ in the equation (Hunt, 1990): $R G R=\frac{\ln (W 2)-\ln (W 1)}{t 2-t 1}$, where $\mathrm{W} 1$ and $\mathrm{W} 2$ are the whole plant fresh weights at times $\mathrm{t} 1$ (at $0 \mathrm{~h}$ of the salt treatment) and $\mathrm{t} 2$ (at $96 \mathrm{~h}$ of the salt treatment). Growth determinations were performed with six replicates and six experiments. All data were statistically analyzed with SAS software (Version 8.2; SAS Institute, Cary, NC) using the $t$ test, and an $\alpha$ value of 0.05 was considered statistically significant.

Total RNA EXTRACTION AND CDNA sYNThesis. Root (the middle part), stem (internode between the third and fourth leaves), and leaf (margins and major veins removed) samples were collected from three different plants harvested from three different plastic containers at $0,6,24,48,72$, and $96 \mathrm{~h}$ of salt treatment. The samples were immediately frozen in liquid nitrogen and stored at $-80^{\circ} \mathrm{C}$ until used for subsequent analysis. The total RNA was extracted from all of the samples using the RNAiso Plus kit (TaKaRa Bio, Shiga, Japan). To remove any contaminating genomic DNA, the RNA was treated with DNase (TaKaRa Bio). The DNase-treated RNA concentration was determined using an ultraviolet spectrophotometer, and the RNA integrity was visualized by $1.2 \%$ agarose gel electrophoresis. For all of the samples, the total RNA $(1 \mu \mathrm{g})$ was converted to cDNA using a Superscript first-strand synthesis system for reverse transcription-polymerase chain reaction (PCR) according to the manufacturer's instructions (TaKaRa Bio). Sample
Bio) and sequenced by Biological Engineering Co., Ltd., Shanghai, China.

The CsaNAPOD coding sequence is $1035 \mathrm{bp}$ and encodes a deduced 344 amino acid sequence, which is identical to the Csa023919 sequence.

Bioinformatics analysis. The Protein-BLAST program at the National Center of Biotechnology Information (NCBI, Bethesda, MD) was used to search for sequence homology. The accession numbers of the amino acid sequences are as follows: $C$. sativus (XP_004151583.1), C. melo (AAR19041.1), Cucurbita pepo (ABF68751.1), Theobroma cacao (EOY09519.1). Multiple sequence alignment was determined using the BioEdit programs. To predict the isoelectric point and molecular weight, ExPasy (Swiss Institute of Bioinformatics, 2005) software was used. Signal peptides of the deduced proteins were determined by Signal-3L (Shen and Chou, 2007). The cell localization of the protein was predicted based on its amino acid sequence by PSORT (Nakai, 2007).

Phylogenetic analysis. All of the POD sequences (Supplementary material) mentioned in the text are available in the peroxidase databases (Laboratoire de Recherche en Sciences Végétales, 2004) and were used to build the phylogenetic tree. Multiple sequence alignment based on the full-length POD proteins was determined using the ClustalW method in MEGA Version 3.1 (Kumar et al., 2004). The phylogenetic trees were 
constructed using the neighbor-joining method. The bootstrap analysis was set at 1000 replicates to evaluate the reliability of the different phylogenetic groups. The tree files were viewed and edited by MEGA 3.1 software.

Quantitative Real-time PCR (QRT-PCR) analysis. To understand the effects of salt stress on CsaNAPOD expression, we treated cucumber seedlings with $50 \mathrm{~mm} \mathrm{NaCl}$ stress for $96 \mathrm{~h}$. The expression of CsaNAPOD in the leaf, stem, and root was determined by quantitative real-time PCR (qRT-PCR) at 0, 6, 24, 48,72 , and $96 \mathrm{~h}$ of treatment. qRT-PCR was performed in a 96well plate format ABI 7300 Real-Time PCR System (Applied Biosystems, Foster City, CA). The reactions $(20 \mu \mathrm{L})$ were conducted using the SuperReal PreMix Plus (SYBR Green) kit (Tiangen Biotech, Beijing, China) according to the manufacturer's protocol. For qRT-PCR, we designed primers specific for $P O D$, forward, 5-TGAGCTGCCTGTTTCTGCATAT-3, reverse, 5-CAAAACAAAAGATGAGCCTACCC-3; for CsaActin, forward, 5-CCGTTCTGTCCCTCTACGCTAGTG3, reverse, 5-GGAACTGCTCTTTGCAGTCTCGAG-3. As an internal standard, the expression level of CsaActin in control and salt-treated cucumber roots was consistent and relatively stable (Du et al., 2010b; Kabała and Kłobus, 2008). The PCR conditions were optimized for each primer set. The PCR amplification was conducted after denaturing the cDNA at $95{ }^{\circ} \mathrm{C}$ for $15 \mathrm{~min}$ followed by 40 cycles of $95{ }^{\circ} \mathrm{C}$ for $10 \mathrm{~s}$, annealing at $58{ }^{\circ} \mathrm{C}$ for $20 \mathrm{~s}$, and extension at $72{ }^{\circ} \mathrm{C}$ for $31 \mathrm{~s}$. The experimental samples, internal standards, and no template controls were run in triplicate. The relative gene expression was quantified using the comparative cycle threshold $(\mathrm{Ct})$ method (referred to as the $2^{-\Delta \Delta \mathrm{CT}}$ method) (Livak and Schmittgen, 2001). All data were statistically analyzed with SAS software (Version 8.2). The $t$ test was used for the statistical calculation of comparison data between the control and salt treatment. Duncan's multiple range test was used for the statistical calculation of the data obtained by comparisons among the three tissues or six time points.

\section{Results}

As shown in Figure 1, compared with the control plants, the plant fresh weight (Fig. 1B), dry weight (Fig. 1C), and RGR (Fig. 1D) of salt-treated plants at $96 \mathrm{~h}$ of treatment were decreased by $36.2 \%, 16.4 \%$, and $64.6 \%$, respectively $(P<0.05)$. This result indicates that salt stress significantly inhibited the growth of the cucumber seedlings.

Sequence analysis showed that the deduced amino acid sequence of CsaNAPOD harbors three conserved domains: the conserved distal heme-binding (B) and the central conserved (D) and proximal heme-binding $(\mathrm{F})$ domains of unknown function (Fig. 2). The CsaNAPOD amino acid sequence also contains eight conserved cysteine residues leading to four disulfide bridges. These characteristics are common in most class III PODs. The analysis of the translated polypeptide also revealed the presence of two putative glycosylation sites and indispensable amino acids required for the heme-binding coordination of $\mathrm{Ca}^{2+}$ ions (Fig. 2).

According to the predicted localization by PSORT, CsaNAPOD may be secreted extracellularly. A predicted 23 amino acid signal peptide was identified by Signal-3L at the $5^{\prime}$ end of the amino acid sequence, which is consistent with an apoplastic protein. CsaNAPOD. The primer sequences were as follows: for CsaNA-

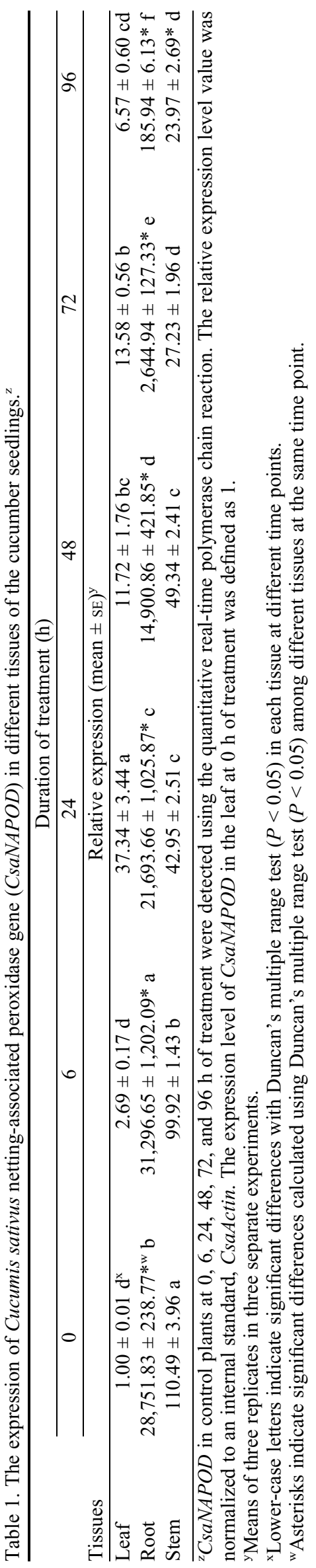




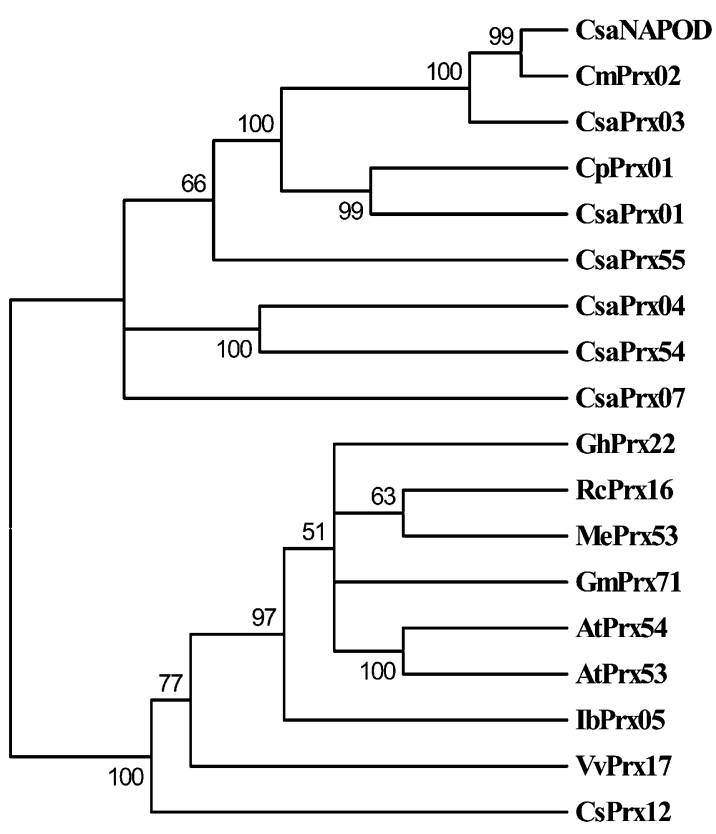

$\begin{array}{llllll}0.25 & 0.20 & 0.15 & 0.10 & 0.05 & 0.00\end{array}$

Fig. 3. Phylogenetic relationships of Cucumis sativus netting-associated peroxidase (CsaNAPOD) with other class III peroxidase (POD) amino acid sequences. The phylogenetic tree file was produced by MEGA 3.1 (Kumar et al., 2004). The bootstrap values were obtained by the neighbor-joining method and indicate the divergence of each branch. The scale indicates the branch length. All the protein sequences (Supplementary material) are available in the peroxidase database (Laboratoire de Recherche en Sciences Végétales, 2004); C. sativus (Csa), Cucumis melo (Cm), Cucurbita pepo (Cp), Gossypium hirsutum (Gh), Ricinus communis (Rc), Manihot esculenta (Me), Glycine max (Gm), Arabidopsis thaliana (At), Ipomoea batatas (Ib), Citrus sinensi (Cs), Vitis vinifera $(\mathrm{Vv})$

Using Expasy software, the theoretical isoelectric point and molecular weight of the deduced CsaNAPOD protein were predicted to be 5.64 and $37.2 \mathrm{kDa}$, respectively. The alignment of the deduced amino acid sequences with homologs from other species showed that CsaNAPOD has the highest similarity to the netting-associated anionic POD of C. melo [AAR19041.1 (93\%)] followed by the PODs of C. pepo [ABF68751.1 (74\%)] and T. cacao [EOY09519.1 (57\%)].

The analysis of the phylogenetic relationships of CsaNAPOD with other class III POD proteins indicated that the POD homologs from the Cucurbitaceae were positioned on a wellsupported branch clearly separated from the PODs present in other families (Fig. 3). The percentage of identity decreased greatly with sequences from Citrus sinensis and other nonCucurbitaceae species. In $A$. thaliana, the closest ortholog was AtPrx 53 with 56\% identity to the CsaNAPOD protein sequence.

The results showed that among the three tested organs, CsaNAPOD was highly expressed in the root with lower expression in the stem and leaf (Table 1). The expression of CsaNAPOD increased within $24 \mathrm{~h}$ and decreased after $48 \mathrm{~h}$ in the leaves of the cucumber seedlings in the control treatment (Fig. 4); however, the overall trend of CsaNAPOD expression declined within the $96 \mathrm{~h}$ of treatment in the roots and stems of the cucumber seedlings. CsaNAPOD in the leaves of the cucumber seedlings was significantly induced after $6 \mathrm{~h}$ of $\mathrm{NaCl}$ treatment. The CsaNAPOD transcript levels were significantly enhanced by 7.9-, 3.9-, 7.2-, 6.2-, and 5.5-fold of control at 6,
24, 48, 72, and $96 \mathrm{~h}$ of treatment, respectively (Fig. 4A). As shown in Figure 4B, compared with the control, salt stress caused a decrease in CsaNAPOD transcripts in the roots of cucumber seedlings after $6 \mathrm{~h}$ of treatment and caused an increase at 48 and $72 \mathrm{~h}$, and no obvious change was observed at 24 and $96 \mathrm{~h}$. The CsaNAPOD transcript level in the stems of cucumber seedlings under $\mathrm{NaCl}$ treatment decreased compared with the control at 48 and $72 \mathrm{~h}$ of treatment, respectively, and no significant change in the transcript level was observed at 6, 24, and $96 \mathrm{~h}$ after salt treatment (Fig. 4C).

\section{Discussion and Conclusions}

Salt stress causes adverse effects on plant growth and productivity (Parida and Das, 2005), and our results showed that cucumber seedling growth was reduced by salt stress (Fig. 1). This result is in agreement with the previous reports regarding salt-treated cucumbers (Du et al., 2010a; Duan et al., 2008; Fan et al., 2012; Shi et al., 2007). Duan et al. (2008) suggested that cucumber seedlings most likely increase the protein level of antioxidant enzymes and the content of osmoticants to enhance resistance to salinity. The molecular mechanism of this process remains unclear; however, it has been well established that any stress condition or significant change in the environment is associated with the up- or downregulation of several hundreds of genes, including PODs (Cheeseman, 2007).

We identified a protein termed NAPOD, which was induced by salt stress in cucumber seedling roots (Du et al., 2010b). In this study, our result showed that this peroxidase belongs to class III PODs. The predicted amino acid sequence of the CsaNAPOD gene was compared with other known class III POD sequences (Fig. 2). The alignment analysis showed that the CsaNAPOD protein harbored conserved structural features and activity sites shared by the majority of the class III PODs, including the predicted N-terminal signal peptide; the highly conserved $\mathrm{B}, \mathrm{D}$, and $\mathrm{F}$ domains essential to enzyme catalysis and protein folding; and eight cysteine residues involved in the formation of four disulfide bridges. Two putative glycosylation sites also found in the $C$. melo CmNAPOD (KerenKeiserman et al., 2004) suggest that modifications of the mature CsaNAPOD occur.

As a result of the recent plant genome project activities, many POD gene sequences or homologous sequences have been identified. Phylogenetic analyses have been conducted for PODs from several plants, including A. thaliana (Tognolli et al., 2002), rice (Zhang et al., 2001), cotton [Gossypium hirsutum (Delannoy et al., 2006)], buffalograss [Buchloe dactyloides (Gulsen et al., 2007)], and apple [Malus sieversii (Gulsen et al., 2010)]. Here, the analysis of the phylogenetic relationships of CsaNAPOD with other class III POD proteins confirmed that CsaNAPOD is a member of the class III POD subfamily. The CsaNAPOD grouped with Cucurbitaceae PODs to form a branch clearly separated from the other species (Fig. 3).

A few $P O D$ genes exhibit organ-dependent expression; however, other $P O D$ genes are active in the entire plant (Tognolli et al., 2002; Welinder et al., 2002). In the $A$. thaliana genome, 73 class III POD genes have been annotated, 65 of which were expressed in various tissues, and only three were identified as specific to roots (Valério et al., 2004). Delannoy et al. (2006) reported the expression profiles of 10 cotton class III POD genes (pod1 to pod10) from several different cotton tissues examined. None of the 10 pod genes was found to be 
(A) Leaf

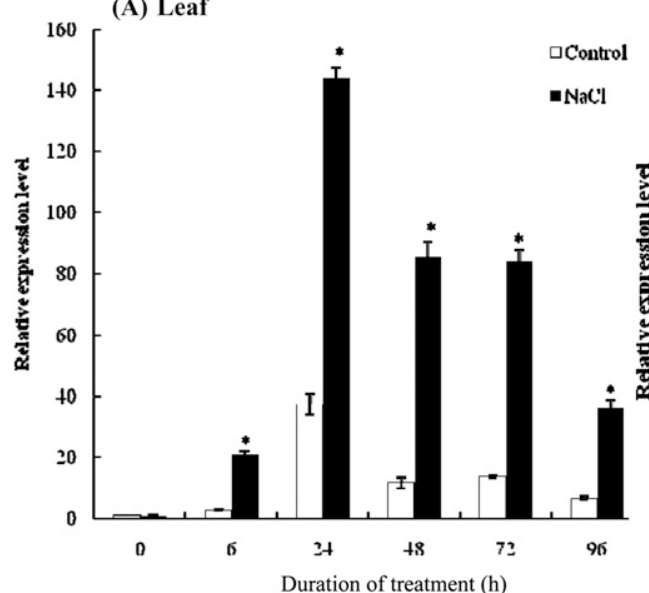

(C) Stem

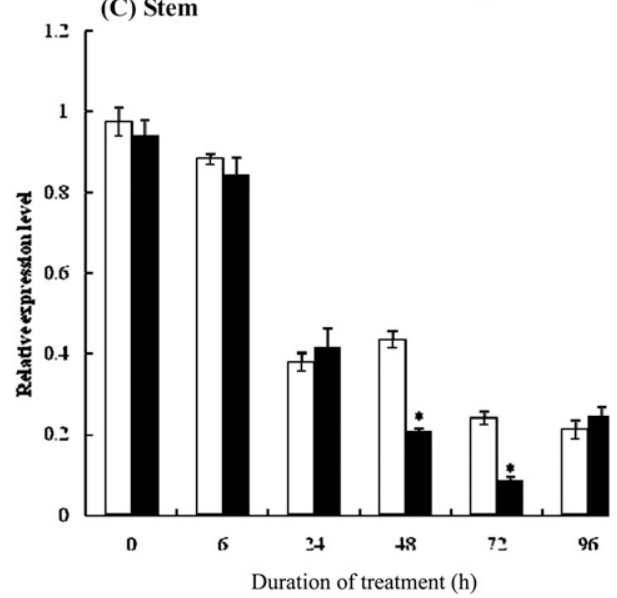

Fig. 4. The effect of salt stress on the expression of Cucumis sativus netting-associated peroxidase gene (CsaNAPOD) in the leaf (A), stem (B), and root (C) of cucumber seedlings. The transcript levels of CsaNAPOD in cucumber seedlings under $\mathrm{NaCl}$ stress after $0,6,24,48,72$, and $96 \mathrm{~h}$ of treatment were detected using the quantitative realtime polymerase chain reaction. Control $=$ control plants; $\mathrm{NaCl}=50 \mathrm{~mm} \mathrm{NaCl}$-treated plants. The relative expression level value was normalized to an internal standard CsaActin. The expression level of CsaNAPOD in control plants at $0 \mathrm{~h}$ of treatment was defined as 1 . Each histogram represents the mean value of three independent experiments, and the vertical bars indicate SE $(\mathrm{n}=3)$. Asterisks indicate the significant differences between control and $\mathrm{NaCl}$ stress at each time point calculated using the $t$ test $(* P<0.05)$. organ-specific. Class III PODs generally do not appear to have a high level of tissue specificity (Tognolli et al., 2002). However, in recent years, class III PODs with organ/tissue specificity were identified (Cosio and Dunand, 2009; Mei et al., 2009). In S. squalidus, a stigma-specific class III POD was identified that may be involved in the defense against pathogens or in pollen-stigma signaling (McInnis et al., 2005). Three PODs are specifically expressed in stigmas but are not found in other floral tissues/organs in A. thaliana (Swanson et al., 2005). In the present study, CsaNAPOD showed no organ specificity and was expressed in each organ evaluated (root, leaf, and stem); however, the transcript abundance of CsaNAPOD was different in these organs. For example, CsaNAPOD demonstrated high expression in roots and lower expression in the leaves and stems (Table 1), suggesting that, at least to a certain extent, the role of CsaNAPOD may be distinct in different organs. The relatively high expression of CsaNAPOD in roots may be used for eliminating excessive ROS generated by salt stress, which suggests that CsaNAPOD may be associated with the most powerful defense against salinity in roots.
PODs have been associated with high salinity stress in a few plants. Ritter et al. (1993) demonstrated a peroxidase mRNA was induced 3fold in the young leaves of saltstressed cotton plants. Kumar et al. (2011) indicated that the expression levels of $C r P r x 3$ and $C r P r x 4$, two new class III POD genes, were downregulated in Catharanthus roseus under salt stress. In addition, the overexpression of the sweetpotato (Ipomoea batatas) swpa4 peroxidase significantly enhanced the salt stress tolerance of tobacco plants [Nicotiana tabacum (Kim et al., 2008)]. In our study, CsaNAPOD protein in cucumber seedling roots was induced at $3 \mathrm{~d}$ under $\mathrm{NaCl}$ stress $(\mathrm{Du}$ et al., 2010b), which is in agreement with the transcript expression in roots at $3 \mathrm{~d}(72 \mathrm{~h})$ after starting $\mathrm{NaCl}$ treatment.

In our study, salt stress greatly upand downregulated CsaNAPOD expression in the leaves and roots of cucumber seedlings, respectively, at $6 \mathrm{~h}$ after the start of treatment, indicating the effects of salt stress in these two organs at the early stage of treatment in the experiment. CsaNA$P O D$ expression in the stem was affected by salt at $48 \mathrm{~h}$ of treatment. Salt stress caused different effects on CsaNAPOD transcript accumulation in the leaves, roots, and stems of cucumber seedlings, suggesting that the response to salt stress was organspecific. The differential expression of CsaNAPOD among different tissues may be considered to dominate the steady-state level of ROS; therefore, neutralizing the toxic effects of salt stress is crucial. Our results indicate that CsaNAPOD expression in leaves and roots is very rapidly induced and suppressed in cucumber seedlings under salt stress, respectively, suggesting that $C s a N A P O D$ in leaves and roots may play an important role in the cucumber plant against salinity. Moreover, the upregulated expression of CsaNAPOD in leaves during the entire experimental period and in the roots at 48 and $72 \mathrm{~h}$ after starting treatment under salt stress may be directly involved in a variety of protective or adaptive biological mechanisms controlling distinct cellular functions under different environmental conditions.

Plant class III PODs have been proposed as key regulators of the level of extracellular $\mathrm{H}_{2} \mathrm{O}_{2}$ and as producers of the extremely reactive hydroxyl ions $(\mathrm{OH} \bullet)$ and hydroperoxyl $(\mathrm{OOH} \bullet)$ radicals (Bolwell et al., 2002), depending on whether the peroxidative or the hydroxylic cycles of the enzyme are operating (Kawano, 2003; Passardi et al., 2005). In this study, the study of salinity-induced CsaNAPOD regulation is an essential issue, directly implicated in the understanding of the biological functions involved in plant defense response. A 
broad spectrum of defense responses includes early and late responsive genes geared toward immediate defense and longlasting defense strategies. Different peroxidase isoenzymes likely play an essential role in the normal development and physiology of the plants during different developmental stages. Regardless of whether or when the expression of the gene was inhibited or increased by salt stress, our observations suggest that CSANAPOD has a potential defense function in cucumber plants against salt stress. PODs are activated in response to stress-related signals of a biotic or abiotic nature and are believed to play key roles in these processes. To better understand the function of peroxidases, further experiments using the overexpression and suppression of each gene in transgenic plants are necessary.

\section{Literature Cited}

Ansari, K.I., S. Walter, J.M. Brennan, M. Lemmens, S. Kessans, A. McGahern, D. Egan, and F.M. Doohan. 2007. Retrotransposon and gene activation in wheat in response to mycotoxigenic and nonmycotoxigenic-associated Fusarium stress. Theor. Appl. Genet. 114:927-937.

Bolwell, G.P., L.V. Bindschedler, K.A. Blee, V.S. Butt, D.R. Davies, S.L. Gardner, C. Gerrish, and F. Minibayeva. 2002. The apoplastic oxidative burst in response to biotic stress in plants: A three component system. J. Expt. Bot. 53:1367-1376.

Cheeseman, J.M. 2007. Hydrogen peroxide and plant stress: A challenging relationship. Plant Stress 1:4-15.

Chinese Academy of Agricultural Sciences. 2009. Cucumber genome database. 24 Feb. 2014. <http://cucumber.genomics.org.cn/page/ cucumber/index.jsp $>$.

Cosio, C. and C. Dunand. 2009. Specific functions of individual class III peroxidase genes. J. Expt. Bot. 60:391-408.

Csiszár, J., Á. Gallé, E. Horváth, P. Dancsó, M. Gombos, Z. Váry, L. Erdei, J. Györgyey, and I. Tari. 2012. Different peroxidase activities and expression of abiotic stress-related peroxidases in apical root segments of wheat genotypes with different drought stress tolerance under osmotic stress. Plant Physiol. Biochem. 52:119-129.

De Gara, L. 2004. Class III peroxidases and ascorbate metabolism in plants. Phytochem. Rev. 3:195-205.

Delannoy, E., P. Marmey, A. Jalloul, H. Etienne, and M. Nicole. 2006. Molecular biology and physiology, molecular analysis of class III peroxidases from cotton. J. Cotton Sci. 10:53-60.

Du, C.-X., H.-F. Fan, S.-R. Guo, and T. Tezuka. 2010a. Applying spermidine for differential responses of antioxidant enzymes in cucumber subjected to short-term salinity. J. Amer. Soc. Hort. Sci. 135:18-24.

Du, C.-X., H.-F. Fan, S.-R. Guo, T. Tezuka, and J. Li. 2010 b. Proteomic analysis of cucumber seedling roots subjected to salt stress. Phytochemistry 71:1450-1459.

Duan, J., J. Li, S. Guo, and Y. Kang. 2008. Exogenous spermidine affects polyamine metabolism in salinity-stressed Cucumis sativus roots and enhances short-term salinity tolerance. J. Plant Physiol. 165:1620-1635.

Fan, H.F., C.X. Du, and S.R. Guo. 2012. Effect of nitric oxide on proline metabolism in cucumber seedlings under salinity stress. J. Amer. Soc. Hort. Sci. 137:127-133.

Gerchikov, N., A. Keren-Keiserman, R. Perl-Treves, and I. Ginzberg. 2008. Wounding of melon fruits as a model system to study rind netting. Sci. Hort. 117:115-122.

Gulsen, O., S. Kaymak, S. Ozongun, and A. Uzun. 2010. Genetic analysis of Turkish apple germplasm using peroxidase gene-based markers. Sci. Hort. 125:368-373.

Gulsen, O., R.C. Shearman, T.M. Heng-Moss, N. Mutlu, D.J. Lee, and G. Sarath. 2007. Peroxidase gene polymorphism in buffalograss and other grasses. Crop Sci. 47:767-772.
Hunt, R. 1990. Basic growth analysis: Plant growth analysis for beginners. Unwin Hyman, London, UK.

International Brachypodium Initiative. 2010. Genome sequencing and analysis of the model grass Brachypodium distachyon. Nature 463:763-768.

Kabała, K. and G. Kłobus. 2008. Modification of vacuolar proton pumps in cucumber roots under salt stress. J. Plant Physiol. $165: 1830-1837$

Kawano, T. 2003. Roles of the reactive oxygen species-generating peroxidase reactions in plant defense and growth induction. Plant Cell Rpt. 21:829-837.

Keren-Keiserman, A., Z. Tanami, O. Shoseyov, and I. Ginzberg. 2004. Peroxidase activity associated with suberization processes of the muskmelon (Cucumis melo) rind. Physiol. Plant. 121:141-148.

Kim, Y.H., C.Y. Kim, W.K. Song, D.S. Park, S.Y. Kwon, H.S. Lee, J.W. Bang, and S.S. Kwak. 2008. Overexpression of sweetpotato swpa4 peroxidase results in increased hydrogen peroxide production and enhances stress tolerance in tobacco. Planta 227:867-881.

Kim, Y.H., S. Lim, S.H. Han, J.C. Lee, W.K. Song, J.W. Bang, S.Y. Kwon, H.S. Lee, and S.S. Kwak. 2007. Differential expression of 10 sweetpotato peroxidases in response to sulfur dioxide, ozone, and ultraviolet radiation. Plant Physiol. Biotechnol. 45:908-914.

Kumar, S., M. Jaggi, J. Taneja, and A.K. Sinha. 2011. Cloning and characterization of two new class III peroxidase genes from Catharanthus roseus. Plant Physiol. Biochem. 49:404-412.

Kumar, S., K. Tamura, and M. Nei. 2004. MEGA3: Integrated software for molecular evolutionary genetics analysis and sequence alignment. Brief. Bioinform. 5:150-163.

Kumari, M., J.T. Gregory, and K.D. Michael. 2008. Transcriptomic responses to aluminum stress in roots of Arabidopsis thaliana. Mol. Genet. Genomics 279:339-357.

Laboratoire de Recherche en Sciences Végétales. 2004. The peroxidase databases. 24 Feb. 2014. $<$ https://peroxibase.toulouse.inra.fr/>.

Livak, K.J. and T.D. Schmittgen. 2001. Analysis of relative gene expression data using real-time quantitative PCR and the $2^{-\Delta \Delta \mathrm{CT}}$ method. Methods 25:402-408.

Martinez, A.T., M. Speranza, F.J. Ruiz-Duenas, P. Ferreira, S. Camarero, F. Guillen, M.J. Martinez, A. Gutierrez, and J.C. del Rio. 2005. Biodegradation of lignocellulosics: Microbial, chemical, and enzymatic aspects of the fungal attack of lignin. Intl. Microbiol. 8:195-204.

McInnis, S.M., L.M. Costa, J.F. Gutiérrez-Marcos, C.A. Henderson, and J. Hiscock. 2005. Isolation and characterization of a polymorphic stigma-specific class III peroxidase gene from Senecio squalidus L. (Asteraceae). Plant Mol. Biol. 57:659-677.

Mei, W., Y. Qin, W. Song, J. Li, and Y. Zhu. 2009. Cotton GhPOXI encoding plant class III peroxidase may be responsible for the high level of reactive oxygen species production that is related to cotton fiber elongation. J. Genet. Genomics 36:141-150.

Mohammadi, M., N.N. Kav, and M.K. Deyholos. 2008. Transcript expression profile of water-limited roots of hexaploid wheat (Triticum aestivum 'Opata'). Genome 51:357-367.

Nakai, K. 2007. PSORT. 24 Feb. 2014. <http://psort.hgc.jp/>.

Parida, A.K. and A.B. Das. 2005. Salt tolerance and salinity effects on plants: A review. Ecotoxicol. Environ. Saf. 60:324-349.

Passardi, F., C. Cosio, C. Penel, and C. Dunand. 2005. Peroxidases have more functions than a Swiss army knife. Plant Cell Rpt. 24:255265.

Passardi, F., D. Longet, C. Penel, and C. Dunand. 2004. The class III peroxidase multigenic family in rice and its evolution in land plants. Phytochemistry 65:1879-1893.

Ritter, D., R.D. Allen, N. Trolinder, D.W. Hughes, and G.A. Galau. 1993. Cotton cotyledon cDNA encoding a peroxidase. Plant Physiol. 102:1351.

Severino, F.E., M. Brandalise, C.S. Costa, S.R.S. Wilcken, M.P. Maluf, W. Gonçalves, and I.G. Maia. 2012. CaPrx, a Coffea arabica gene encoding a putative class III peroxidase induced by root-knot nematode infection. Plant Sci. 35-42:191-192. 
Shen, H.B. and K.C. Chou. 2007. Signal-3L. 24 Feb. 2014. <http:// www.csbio.sjtu.edu.cn/bioinf/Signal-3L/>.

Shi, Q., F. Ding, X. Wang, and M. Wei. 2007. Exogenous nitric oxide protect cucumber roots against oxidative stress induced by salt stress. Plant Physiol. Biochem. 45:542-550.

Shigeoka, S., T. Ishikawa, M. Tamoi, Y. Miyagawa, T. Takeda, Y. Yabuta, and K. Yoshimura. 2002. Regulation and function of ascorbate peroxidase isoenzymes. J. Expt. Bot. 53:1305-1319.

Smulevich, G., C. Jakopitsch, E. Droghetti, and C. Obinger. 2006. Probing the structure and bifunctionality of catalase-peroxidase (KatG). J. Inorg. Biochem. 100:568-585.

Swanson, R., T. Clark, and D. Preuss. 2005. Expression profiling of Arabidopsis stigma tissue identifies stigma-specific genes. Sex. Plant Reprod. 18:163-171.

Swiss Institute of Bioinformatics. 2005. ProtParam. 24 Feb. 2014. $<$ http://web.expasy.org/protparam/>.
Tognolli, M., C. Penel, H. Greppin, and P. Simon. 2002. Analysis and expression of the class III peroxidase large gene family in Arabidopsis thaliana. Gene 238:129-138.

Valério, L., M. De Meyer, C. Penel, and S. Dunand. 2004. Expression analysis of the Arabidopsis peroxidase multigene family. Phytochemistry 65:1331-1342.

van Huystee, R.B. 1987. Some molecular aspects of plant peroxidase biosynthetic studies. Annu. Rev. Plant Physiol. 38:205219.

Welinder, K.G., A.F. Justesen, I.V. Kjaersgard, R.B. Jensen, S.K. Rasmussen, H.M. Jespersen, and L. Duroux. 2002. Structural diversity and transcription of class III peroxidases from Arabidopsis thaliana. Eur. J. Biochem. 269:6063-6081.

Zhang, L., S.K. Pond, and B.S. Gaut. 2001. A survey of the molecular evolutionary dynamics of twenty-five multigene families from four grass taxa. J. Mol. Evol. 52:144-156. 